\title{
Consistency and contrast effects in moral evaluation of euthanasia
}

Štěpán Bahník ${ }^{1 *}$ and Marek A. Vranka ${ }^{2,3}$

${ }^{1}$ The Prague College of Psychosocial Studies, Hekrova 805, Prague, 149 00, Czech Republic. bahniks@seznam.cz

${ }^{2}$ Faculty of Social Sciences, Charles University, Smetanovo nábřeží 6, Prague, 110 01, Czech Republic. marek.vranka@fsv.cuni.cz

${ }^{3}$ Faculty of Arts, Charles University, nám. J. Palacha 2, Prague, 116 38, Czech Republic. marek.vranka@ff.cuni.cz

* Corresponding author

\begin{abstract}
Euthanasia is a highly controversial topic. One of the arguments against legalisation of euthanasia is that it would lead to an attitudinal slippery slope effect; that is, a shift in attitudes toward euthanasia even toward cases which were not legalised. The present study tested a possible mechanism which may lead to such shift in two experiments. Participants judged morality of euthanasia in two hypothetical scenarios describing patients requesting euthanasia. We found that participants who first evaluated a case of a non-terminally ill patient suffering from fatigue afterward considered euthanasia for a terminally ill patient suffering from pain more morally right than participants who evaluated euthanasia in the latter case first. Furthermore, we found that presenting the case of the patient suffering from fatigue before asking about attitudes toward legality of euthanasia led participants to oppose it more. The study suggests that public's expressed attitudes toward legality of euthanasia might be easily influenced by a choice of illustrative examples. However, the change in attitudes predicted by the slippery slope effect was not observed.
\end{abstract}

\section{Keywords}

euthanasia, morality, slippery slope effect, order effect 
Euthanasia ${ }^{1}$ is legalised in an increasing number of countries (Emanuel, Onwuteaka-Philipsen, Urwin, \& Cohen, 2016). One might assume that this development reflects true attitude change of majority of public regarding euthanasia. However, based on previous empirical studies, it seems that people's expressed attitudes are often easily malleable (Wilson \& Hodges, 1992). Expressed attitudes can be influenced by accidental situational factors such as question wording (Schwarz, 2007), current affective state (Fedorikhin \& Cole, 2004; Loewenstein, 1996), or by people's desire to appear consistent (Andrade \& Ariely, 2009; Guadagno \& Cialdini, 2010). For example, people's expressed attitudes can be sometimes reversed by telling them they had previously reported an opposing view (Hall, Johansson, Tärning, Sikström, \& Deutgen, 2010) or by changing an order in which they are asked to judge given issues (Petrinovich \& O'Neill, 1996). Striving for consistency can mold judgments regarding moral issues in general (Vranka \& Bahník, 2016) as well as judgments regarding life and death in particular: when people judge a series of trolley-dilemmas (i.e., when they decide whether it is right or wrong to kill one person in order to save many), their judgments depend on the order in which the dilemmas are presented. When a case in which the sacrifice is considered morally right by a majority of people is presented first, people are subsequently more likely to say that the sacrifice in a more ambiguous situation is also right (O'Hara, Sinnott-Armstrong, \& Sinnott-Armstrong, 2010; Petrinovich \& O’Neill, 1996; Wiegmann, Okan, \& Nagel, 2012).

This seeming malleability of attitudes forms a basis for concern that legalisation of euthanasia may have unintended negative consequences: While euthanasia may be initially intended for use in specific cases of terminally ill adults with unmanageable physical pain, the worry is that the scope of use of euthanasia will broaden as a result of its legalisation (Radbruch et al., 2016). Such slippery slope arguments posit that the initial requirement of terminal illness may be loosened and euthanasia might be used to end lives of people who are not terminally ill, such as people who are simply tired of life, or even people who are not able to express their wish to die, such as babies or people in coma (nonvoluntary euthanasia), or - in an extreme case - lives of people who are able to express their wish to die, but did not (involuntary euthanasia).

Slippery slope effects may occur after legalisation of euthanasia in various ways (Volokh, 2003). One possibility is that people do not perceive, or are not willing to act against, small changes of conditions required for a legal use of euthanasia. For example, if euthanasia is initially legal only for adults older than 18 years, people may not act if this legal limit shifts gradually downward even if they would have opposed the lower final legal age of euthanasia if it had been proposed initially. Another reason for the slippery slope effect might be that arguments previously employed for justification of euthanasia in more circumscribed cases can be later used for its

\footnotetext{
${ }^{1}$ Active euthanasia is defined as "intentionally administering medications or other interventions to cause the patient's death [...]" with voluntary active euthanasia specifically limited to "[...] at the patient's explicit request and with full informed consent" (Emanuel, 1994, p. 1891). Some other possible definitions of euthanasia also specify the patient's condition (e.g., World Health Organization, 2004, p. 25) and some of the cases of euthanasia within the scope of definition used by Emanuel would fall outside of the scope of the definition by WHO.
} 
defense in a wider range of patients: for example, when euthanasia of terminally ill patients is justified by the principle of autonomy, the same principle can be used for justification of euthanasia of non-terminally ill people.

Still another mechanism through which a slippery slope effect may occur is a gradual change of attitudes after legalisation of euthanasia. This attitudinal slippery slope effect, evaluated in the present study, works through change of attitudes after legalisation of euthanasia. An argument for such an effect posits that after judging euthanasia as morally acceptable in one case, people are more likely to judge it as acceptable in other, more ambiguous cases because they strive for consistency in their judgment (Guadagno \& Cialdini, 2010). Because legalisation of euthanasia might make people encounter euthanasia in clear-cut cases more often, it may subsequently lead them to support euthanasia even in cases where they would have opposed it before the legalisation. By changing the attitudes, legalisation of euthanasia in some cases may influence support for legalisation of euthanasia even in cases where it would have been considered untenable before. Although gradual acceptance of clearly unethical use of euthanasia is the main danger implicitly inherent in slippery slope arguments, the necessary condition for existence of the slippery slope effect is that people gradually accept euthanasia in cases which they initially judged as unacceptable, even if others might have considered them ethical all along - the present study experimentally evaluates this prerequisite for the attitudinal slippery slope effect.

Currently available data from real-life settings are limited in their ability to examine whether the slippery slope effect occurs or not. For example, data from Belgium show that the prevalence of euthanasia increased between two timepoints after its legalisation. The increase was associated both with a higher number of euthanasia requests and with a higher proportion of requests actually granted (Dierickx, Deliens, Cohen, \& Chambaere, 2015). Even though Lerner and Caplan (2015) interpreted this trend as an occurrence of the slippery slope effect, such interpretation is questionable, because the described increase by itself does not necessarily imply greater acceptance of euthanasia in cases in which it was considered unacceptable before. Other data show that people were more likely to view euthanasia justifiable after its legalisation in Belgium in comparison to the time before legalisation. Even though the change of attitudes in Belgium may be seen as evidence for the slippery slope effect, the attitudes changed during the same time period also in some countries where euthanasia was not legalised and they did not change in the Netherlands where euthanasia was also legalised during that time (Cohen, Van Landeghem, Carpentier, \& Deliens, 2012). The change in attitudes in Belgium cannot be therefore unequivocally ascribed to the effect of legalisation of euthanasia and accepted as evidence of existence of the slippery slope effect.

Other studies explored the possibility of slippery slope effects by studying the perception of acceptability of euthanasia in hypothetical scenarios. For example, Raijmakers et al. (2013) showed that some Dutch people agreed with euthanasia in a hypothetical case even if the person was only tired of living, which was not a legal reason for euthanasia in the Netherlands. Similarly, a majority of Dutch pediatricians said they would be willing to administer lethal drugs 
even to children who are younger than is required under the current Dutch euthanasia law (Vrakking et al., 2005). These studies show that people may find euthanasia acceptable even in cases where it is not legal. Nevertheless, they do not actually show evidence for slippery slope effects because attitudes measured in them did not have to be necessarily caused by legalisation of euthanasia. A study by Feltz (2015) showed that people judge voluntary euthanasia more acceptable than nonvoluntary euthanasia and that voluntariness has much stronger influence on acceptability of euthanasia than other factors. Even though Feltz argues that this finding provides evidence against slippery slope arguments, the sole fact that people distinguish between voluntary and nonvoluntary euthanasia does not necessarily mean that people's attitudes toward nonvoluntary euthanasia or voluntary euthanasia in a wider range of cases would stay the same after legalisation of voluntary euthanasia.

Given that it is not possible to manipulate legalisation of euthanasia experimentally, any study on the topic can provide only limited evidence for or against existence of the slippery slope effect. It is therefore necessary to study the question with a variety of methods that each may help to understand the topic better despite its limitations. The current study evaluated the attitudinal slippery slope effect by giving participants two hypothetical scenarios which presented cases of incurably ill patients requesting euthanasia who differed in the amount and nature of their suffering. Based on previous findings, we assumed that participants would believe that ending the patient's life would be more morally right in the scenario presenting a case of a terminally ill patient in excruciating pain than in the scenario presenting a case of a patient with fatigue and troubles moving whose condition was not life threatening (Emanuel, 2002). Following the logic of the attitudinal slippery slope effect, we hypothesized that people would be more likely to consider euthanasia as morally right for the fatigued patient if they had accepted euthanasia for the patient in pain before than if they judged morality of euthanasia for the fatigued patient first. Similarly, we expected that participants would be more likely to condemn euthanasia for the patient in pain if they had answered before that euthanasia was morally wrong for the fatigued patient than if they judged morality of euthanasia for the patient in pain first (O'Hara, et al., 2010; Petrinovich \& O’Neill, 1996 ). Given that people often form their attitudes toward legalisation of euthanasia based on its moral perception (Kinsella \& Verhoef, 1993), we expected that the possible order effects on moral evaluation would influence attitudes toward legality of euthanasia as well.

\section{Study 1}

\section{Methods}

Preregistration, materials, data, and R code used for analysis for both studies can be found on https://osf.io/ddnem/. 


\section{Participants and design}

We put a human intelligence task (HIT) on MTurk for 300 US workers. Due to an insufficient check of the data at the time of the submission of participants' work, we obtained data only from 239 participants while the remaining workers claimed the participant fee, but did not submit any responses. We further excluded 13 participants who filled the study twice based on their IP addresses and 5 participants whose data contained missing values. We conducted analysis with the data from the remaining 221 participants.

All participants were given two scenarios describing a hypothetical situation where a patient requests euthanasia. The order of presentation of the scenarios was randomly determined for each participant and the scenarios were presented successively, on different screens. After answering questions relating to the scenarios, participants were asked whether euthanasia should be legal.

During the time of the study, euthanasia in the form of a physician-assisted suicide was legalized in five US states (California, Montana, Oregon, Vermont, and Washington). We did not obtain the information about the state of residence of participants. However, the questions used in the present study were posed in such a manner that the legal status of euthanasia should not have played a role in their interpretation. Furthermore, administration of the lethal substance by the doctor, which was described in the scenarios, is currently illegal in all US states.

\section{Materials}

The two scenarios used in the experiment described a situation where a patient has an incurable illness that causes him distress. The pain scenario was directly taken from Emanuel (2002). In this scenario, the patient is terminally ill and suffers from excruciating pain which cannot be controlled by painkillers or other means:

A patient has an incurable metastatic cancer, which invades the bones and causes excruciating pain. Current levels of morphine, nerve blocks, and other treatments are failing to control the pain. Doctors expect the patient to live for no more than four months.

The fatigue scenario was created from the pain scenario by changing the amount and nature of the distress in order to make the patient's condition much less serious. In the fatigue scenario, the patient is not terminally ill and suffers from fatigue and difficulty moving limbs:

A patient has an incurable illness that affects muscles and tendons. The illness is not life-threatening and the patient does not feel any significant pain. However, he can move his limbs only with great difficulty and feels enormous fatigue all the time. He feels that his life has no meaning anymore and concludes that there is no point in continuing living like this. 
After reading each scenario, participants were asked: "In this case, would it be morally right, upon request from the patient, for the doctor to administer intravenous drugs, such as potassium chloride, to intentionally end the patient's life?" and answered using a 6-point scale ranging from 1 (completely morally right) to 6 (completely morally wrong).

Finally, participants answered whether they believed that voluntary euthanasia should be legal using a 5-point scale ranging from 1 (definitely should be illegal) to 5 (definitely should be legal). The answer to this question served as a secondary dependent variable. We hypothesized that participants might be more likely to agree with legalization if they were given the pain scenario first than if they were presented with the fatigue scenario first.

\section{Results and discussion}

We conducted the analysis of perceived morality of euthanasia in the two scenarios using mixedeffect models with ratings of morality as the dependent variable, scenario, order ${ }^{2}$, and their interaction as predictors, and with a random intercept for participants. Figure 1 and Table 1 display the results. As expected, euthanasia was considered less morally wrong in the pain scenario than in the fatigue scenario, $t(219.0)=6.88, p<.001, b=0.99,95 \%$ CI [0.71, 1.27]. While $44 \%$ of participants judged euthanasia in the fatigue scenario as more wrong than in the pain scenario, only $9 \%$ of participants considered euthanasia in the fatigue scenario as less wrong. Contrary to our prediction, participants evaluating the pain scenario first judged euthanasia on average more morally wrong than participants evaluating the fatigue scenario first, $t(306.2)=2.18, p=.03, b=0.51,95 \%$ CI $[0.05,0.97]$. The interaction between the scenario and order effects suggested that the effect of order might have been stronger for the pain scenario than for the fatigue scenario, $t(219.0)=-1.85, p=.07, b=-0.36,95 \%$ CI $[-0.74,0.02]$, but the result was not statistically significant. The nature of the interaction can be seen from separate analyses of the two scenarios - the ratings of morality of euthanasia in the fatigue scenario did not differ between groups, $t(219)=-0.64, p=.52, d=-0.09,95 \% \mathrm{CI}[-0.35,0.18]$, but they differed in the pain scenario, $t(219)=-2.21, p=.03, d=-0.30,95 \%$ CI $[-0.57,-0.03]$.

The wrongness ratings correlated highly between the two scenarios, $r(219)=.65,95 \%$ CI $[.57$, $.72], p<.001$, and there was also a strong negative correlation between the attitude toward legality of euthanasia and wrongness ratings in both the fatigue, $r(218)=-.62,95 \% \mathrm{CI}[-.69$, $.53], p<.001$, and pain scenario, $r(218)=-.74,95 \%$ CI $[-.80,-.68], p<.001$.

The order of scenarios also affected answers to the legality question in the opposite direction than we had predicted. Participants were more likely to answer that euthanasia should be legal when they were given the fatigue scenario first, $t(218)=2.16, p=.03, d=0.29,95 \%$ CI [0.02,

\footnotetext{
${ }^{2}$ Based on the slippery slope argument we predicted that participants evaluating the pain scenario first (pain-fatigue group) should evaluate the fatigue scenario as less wrong and that participants evaluating the fatigue scenario first (fatigue-pain group) should evaluate the pain scenario as more wrong. These two order effects combined should result in generally higher wrongness ratings for the fatigue-pain group, which is tested by the predictor order.
} 
0.56] (see Figure 2).

\begin{tabular}{llllc}
\multicolumn{5}{c}{ Scenario } \\
Condition & Pain & Fatigue & Both & Legality \\
fatigue-pain & $2.38(1.60)$ & $3.37(1.67)$ & $2.88(1.71)$ & $3.88(1.24)$ \\
pain-fatigue & $2.89(1.79)$ & $3.52(1.81)$ & $3.21(1.82)$ & $3.48(1.43)$ \\
both & $2.67(1.72)$ & $3.46(1.75)$ & $3.06(1.78)$ & $3.66(1.36)$
\end{tabular}

Table 1: Results of Study 1. The table shows mean ratings of wrongness of euthanasia and of approval of legality of euthanasia (with standard deviations in parentheses).

Pain scenario

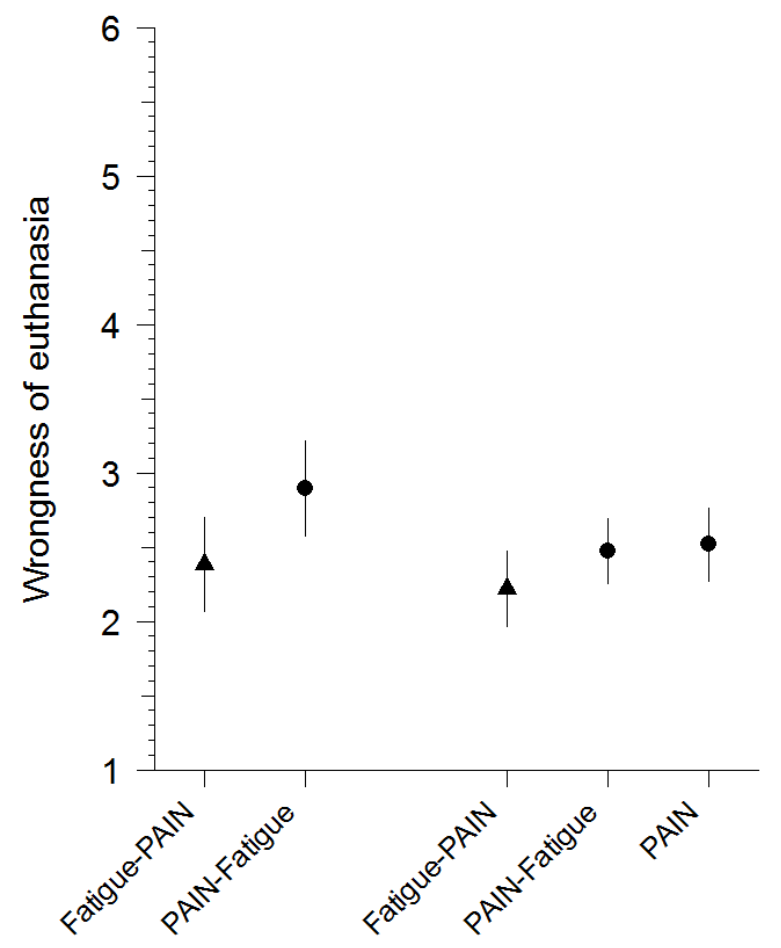

Study 1
Study 2

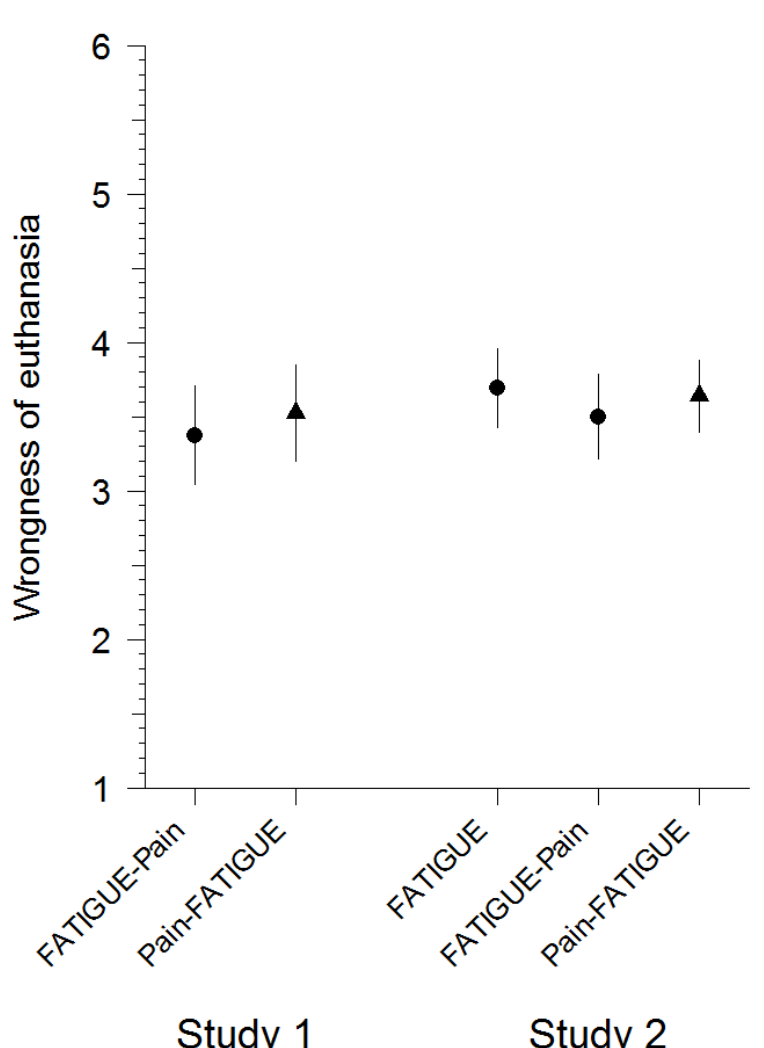

Study 1

Fatigue scenario

Study 2

Figure 1: Evaluation of wrongness of euthanasia in Study 1 and 2. The figure shows average ratings of wrongness of euthanasia in the pain scenario (left) and in the fatigue scenario (right) separately for each group. It is possible to see that euthanasia in the pain scenario was judged to be less wrong by participants who were given the pain scenario second (triangles in the left graph) than those who were given the pain scenario first (circles in the left graph). Evaluation of euthanasia in the fatigue scenario did not differ consistently based on the order of presentation of the two scenarios. The error bars show $95 \%$ confidence intervals for the mean. 


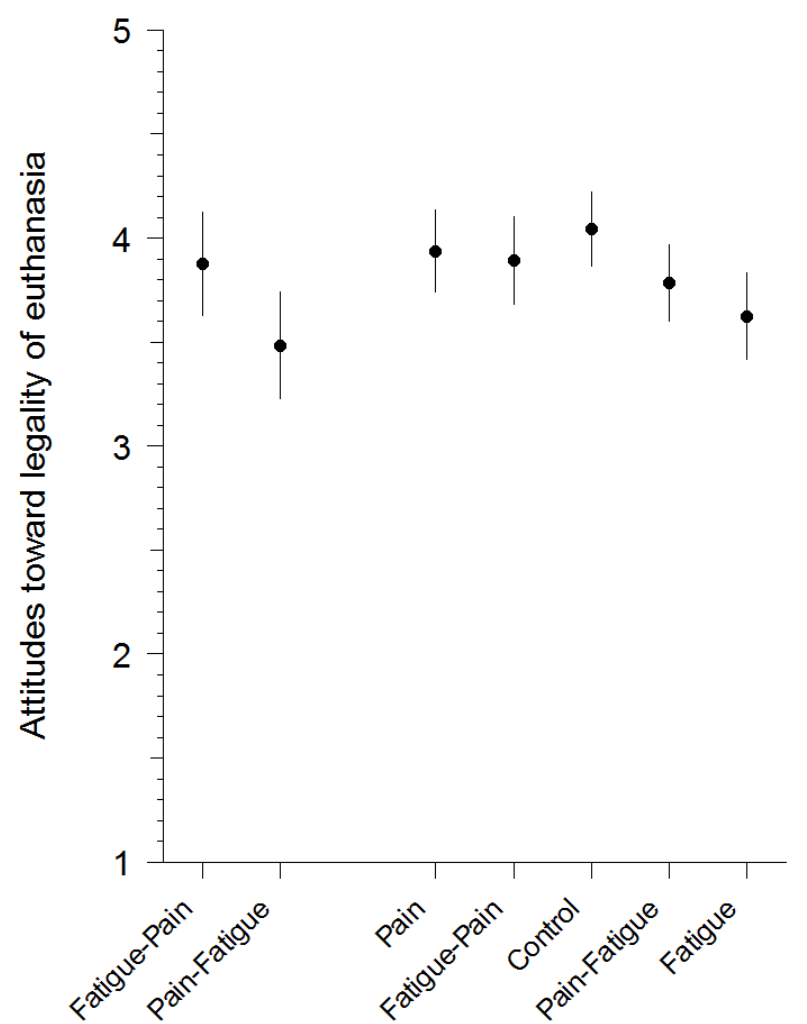

Study 1

Study 2

Figure 2: Attitudes toward legality of euthanasia in Study 1 and 2. The figure shows average ratings of whether euthanasia should be legal separately for each group. Participants who were given the pain scenario directly before answering the question about legality were more likely to answer that euthanasia should be legal than participants given the fatigue scenario directly before the question. The error bars show $95 \%$ confidence intervals for the mean.

One possible explanation for the observed effects being in the opposite direction than we expected is that participants contrasted the second scenario with the first one instead of attempting to be consistent in their judgments (Schwarz \& Bless, 1991). People generally considered euthanasia in the pain scenario as more morally right than euthanasia in the fatigue scenario, and so those judging the scenario with a patient in excruciating pain as second could have considered it even less wrong when compared with the less serious case judged a moment before. By the same token, those who considered the fatigue scenario as second should have seen it as more wrong when compared to the pain scenario; however, the results suggest that the order did not significantly affect the evaluation of the fatigue scenario. This could be caused by the pain scenario being more prototypical of situations in which people usually consider euthanasia (Emanuel et al., 2016). If that is the case, even people who judged the fatigue scenario first might have implicitly compared it with the prototypical situation which is similar to the pain scenario. 
The question whether euthanasia should be legal could have been answered based on the perceived wrongness of euthanasia in the second, more recent scenario. According to this explanation, presenting the fatigue scenario first and the pain scenario second should result in more positive attitude toward legality of euthanasia than if only the pain scenario was presented since presentation of the fatigue scenario led to more positive attitude toward euthanasia in the pain scenario (possibly due to a contrast effect).

Alternatively, participants could have formed their opinion on euthanasia from both presented scenarios and the last one they saw influenced their opinion the most (a recency effect). If that was the case, we would expect that participants given only one scenario would show the same or a larger effect of the scenario presented just before answering the legality question than participants given both scenarios.

Study 2 aimed to replicate and elucidate the results of Study 1. To explain the process which drove the effect in the first study, we gave some participants only one of the scenarios before expressing their attitudes toward legality of euthanasia. Furthermore, some participants were given only the legality question so that it was possible to find out which previously presented scenarios influenced the answers to the legality question and in which direction.

\section{Study 2}

\section{Methods}

We put a HIT on MTurk for 800 US workers and 802 participants completed the study. ${ }^{3}$ We used the same two hypothetical scenarios presenting cases of patients requesting euthanasia as in the first study. However, we added three more experimental conditions in the present study. Participants were therefore randomly divided in five groups. The first two groups (fatigue-pain and pain-fatigue) corresponded to the two groups in Study 1 - they were given one of the two scenarios first and the other one afterward. Then, they answered the question about whether euthanasia should be legal. Another two groups (fatigue and pain) were given just one of the scenarios and then answered the question about legality of euthanasia. As in Study 1, after each scenario we asked participants to evaluate wrongness of euthanasia in the given case. The final group (control) was given only the question about whether euthanasia should be legal. Wording of the scenarios and questions was the same as in Study 1.

\footnotetext{
${ }^{3}$ Participation in both studies was anonymous. Therefore, it is possible that some of the participants took part in both studies. Using a recent estimate by Stewart et al. (2015) that an average laboratory samples out of about 7300 MTurk workers, approximately 33 participants took part in both our studies. A recent study (Chandler, Paolacci, Peer, Mueller, \& Ratliff, 2015) found reduced effect sizes when the same experiment was given twice to the same participants. However, given that the possible nonnaïveté of participants is likely only to reduce the effects studied in the present study and that the expected number of participants who took part in both studies is relatively low, we do not consider participants' nonnaïveté a significant problem.
} 


\section{Results and discussion}

To evaluate replicability of the results of Study 1, we first analyzed only the two groups corresponding to the groups used in Study 1. Figure 1 and Table 2 display the results. We used the same mixed-effect model as in Study 1. Participants again judged euthanasia in the fatigue scenario as more morally wrong than euthanasia in the pain scenario, $t(310.0)=10.53, p<.001$, $b=1.28,95 \%$ CI $[1.04,1.52]$. Most participants (63\%) considered euthanasia in the fatigue scenario as more wrong than in the pain scenario, whereas only $7 \%$ of participants considered euthanasia in the fatigue scenario as less wrong. Participants in the pain-fatigue group again judged euthanasia as more morally wrong than participants in the fatigue-pain group, but the effect was somewhat weaker and statistically nonsignificant this time, $t(464.1)=1.43, p=.15, b$ $=0.26,95 \%$ CI $[-0.09,0.60]$. The interaction between the scenario and order of presentation was not statistically significant, $t(310.0)=-0.71, p=.48, b=-0.12,95 \%$ CI [-0.44, 0.20], but it was again in the same direction as in the first study. That is, the effect of presentation order was somewhat larger for the pain scenario than for the fatigue scenario. When the results of both studies were combined meta-analytically, the effect of order, $z=2.47, p=.01$, was statistically significant, but its interaction with the scenario effect was not, $z=1.72, p=.09$.

\begin{tabular}{llllc}
\multicolumn{5}{c}{ Scenario } \\
Condition & Pain & Fatigue & Both & Legality \\
fatigue & - & $3.69(1.72)$ & - & $3.62(1.35)$ \\
fatigue-pain & $2.22(1.52)$ & $3.50(1.69)$ & $2.86(1.73)$ & $3.89(1.26)$ \\
control & - & - & - & $4.04(1.17)$ \\
pain-fatigue & $2.48(1.46)$ & $3.64(1.60)$ & $3.06(1.64)$ & $3.78(1.23)$ \\
pain & $2.52(1.56)$ & - & - & $3.94(1.26)$ \\
all & $2.41(1.51)$ & $3.62(1.67)$ & - & $3.85(1.26)$
\end{tabular}

Table 2: Results of Study 2. The table shows mean ratings of wrongness of euthanasia and of approval of legality of euthanasia (with standard deviations in parentheses).

In order to increase statistical power, we next analyzed the order effect for the two scenarios separately, but including also the groups given only one scenario (i.e., comparing, for example, the rating of the fatigue scenario in the pain-fatigue group with its ratings in the fatigue and fatigue-pain groups which were both evaluating it first). The analysis showed no difference in wrongness judgments of the fatigue scenario regardless of whether the fatigue scenario was presented first or only after the pain scenario, $t(475)=-0.23, p=.82, d=-0.01,95 \%$ CI $[-0.09$, 0.07]. There was a larger, but also not statistically significant, difference in wrongness judgments of the pain scenario, $t(468)=-1.81, p=.07, d=-0.08,95 \%$ CI $[-0.16,0.01]-$ when euthanasia in the pain scenario was evaluated after euthanasia in the fatigue scenario, it was judged as less wrong than when it was evaluated first. The order effect combined across the two studies was statistically significant for the pain scenario, $z=2.82, p=.005$, but not for the fatigue scenario, $z$ $=0.57, p=.57$. 
Again, the wrongness ratings correlated highly between the two scenarios, $r(310)=.58,95 \% \mathrm{CI}$ $[.51, .65], p<.001$, and there was also a strong negative correlation of the perception whether euthanasia should be legal with wrongness ratings in both the fatigue, $r(475)=-.60,95 \% \mathrm{CI}[-$ $.66,-.54], p<.001$, and pain scenario, $r(468)=-.70,95 \%$ CI $[-.75,-.65], p<.001$.

The effect of the presentation order on the legality question analyzed with the fatigue-pain and pain-fatigue groups was not statistically significant in the present study, $t(310)=0.76, p=.45, d$ $=0.04,95 \%$ CI $[-0.06,0.14]$. The effect on the legality question combined across the two studies was also not statistically significant, $z=1.75, p=.08$. The effect found in the first study was therefore not replicated.

In line with the pre-registration of Study 2, the question about the legality of euthanasia, shared among all groups of participants, was analyzed as the primary variable of interest. Namely, we tested whether the effect of order on the legality question was caused by a contrast effect or by a recency effect of the second scenario. The first explanation predicted that participants given only the pain scenario would be less likely to answer that euthanasia should be legal than those who had been presented the fatigue scenario before. Presentation of the pain scenario before the fatigue scenario would on the other hand decrease the ratings of whether euthanasia should be legal. The second explanation predicted that the answers to the legality question would be influenced by the last presented scenario. We tested these predictions with a regression using the answer to the legality question as the dependent variable. The first explanation was tested by an independent variable coded as 0.5 for the fatigue and fatigue-pain groups and as -0.5 for the pain and pain-fatigue groups (i.e., depending on the first presented scenario). The second explanation was tested by an independent variable coded as 0.5 for the pain and fatigue-pain groups and as 0.5 for the fatigue and pain-fatigue groups (i.e., depending on the last presented scenario). Participants from the control group were not included in this analysis.

The contrast effect of the first presented scenario was not statistically significant and the rating of legality was in fact in the opposite direction than predicted by the first explanation, $t(631)=-$ $1.01, p=.31, b=-0.10,95 \% \mathrm{CI}[-0.30,0.10]$. On the other hand, the effect of the last presented scenario was statistically significant and consistent with the recency effect explanation, $t(631)=$ $2.07, p=.04, b=0.21,95 \% \mathrm{CI}[0.01,0.41]$. The interaction between the two factors was not statistically significant, $t(631)=0.57, p=.57, b=0.12,95 \%$ CI [-0.28, 0.52]. Therefore, the results suggest that the order effect found in the first study was due to the effect of the second presented scenario. However, it does not seem that this effect is driven by the presentation of the pain scenario. On the contrary, the comparison of the control group with the other groups suggests that the presentation of the fatigue scenario before the legality question makes people more likely to say that euthanasia should not be legal (see Figure 2). That is, participants were more likely to say that euthanasia should be legal in the control group than in the fatigue group, $t(330)=3.01, p=.003, d=0.15,95 \% \mathrm{CI}[0.05,0.25]$, and the pain-fatigue group, $t(337)=1.97$, $p=.05, d=0.10,95 \%$ CI $[0.00,0.20]$. On the other hand, there was no statistically significant 
difference for the fatigue-pain group, $t(305)=1.08, p=.28, d=0.05,95 \%$ CI [-0.04, 0.15], and pain group, $t(323)=0.78, p=.44, d=0.04,95 \%$ CI [-0.06, 0.14].

\section{General discussion}

In the current study, we tested a mechanism that may result in the attitudinal slippery slope effect - when people first consider a situation where euthanasia seems justifiable, they may then perceive euthanasia as morally right even in situations in which they would not have found it justifiable before. However, we did not find the effect. In fact, we found that people evaluated euthanasia in a situation of a terminally ill patient with unmanageable pain as more morally right if they had previously evaluated euthanasia of a non-terminally ill patient suffering from fatigue and movement problems. Furthermore, we found that thinking about particular situations where euthanasia may be used could influence the expressed attitude toward legality of euthanasia. Participants were more likely to say that euthanasia should not be legal if they had thought about euthanasia of a non-terminally ill patient right before evaluating the legality of euthanasia.

While we did not find the order effect consistent with the attitudinal slippery slope effect, the results showing the order effect were equivocal. We found that only presenting the situation of a non-terminally ill patient, where euthanasia seemed less justifiable, influenced perceived wrongness of euthanasia in the case of a terminally ill patient suffering from unmanageable pain, where euthanasia seemed more justifiable. There was no similar effect on evaluation of euthanasia of the non-terminally ill patient suffering from fatigue and with troubles moving. The results of the second study suggested that the order effect was caused by participants trying to use the wrongness scale consistently for evaluation of both scenarios. Participants might have been more restricted in their use of the rating scale if they first evaluated the fatigue scenario where the average answer was around the midpoint of the scale. That is, participants who rated the fatigue scenario as 3 or 4 on a 1-6 wrongness scale would have restricted their possible ratings to 1-2 or 1-3 if they considered euthanasia in the pain scenario more morally right. Participants who rated the pain scenario first and used ratings 2 or 3 would be restricted in their ratings less - to 3-6 or 4-6 if they considered euthanasia in the fatigue scenario more morally wrong. Therefore, the lack of the order effect for participants who were given the pain scenario first is compatible with the explanation that participants were unsure how to map their attitudes to the rating scale in the first scenario and were restricted in their use of the scale when expressing their attitude about the second scenario.

While we did not replicate the effect of the order of scenario presentation on the answers to the question whether euthanasia should be legal, we found that they were influenced by the fatigue scenario if it was considered just before responding to the question. This suggests that it may be possible to influence expressed attitudes toward legality of euthanasia by presenting people situations that they might not be likely to consider (Elliott, 1993). This may make such a case more accessible to people when they later think whether euthanasia should be legal. On the other hand, we found no effect of presentation of a more prototypical euthanasia case of a terminally 
ill patient suffering from unmanageable pain, which might have been easily accessible even without the presentation. Similar accessibility effects have been found in other domains, such as evaluation of life satisfaction (Strack, Martin, \& Schwarz, 1988) or evaluation of one's traits (Schwarz et al., 1991).

The present study has several limitations. First, we studied evaluations of hypothetical scenarios presenting euthanasia cases. While previous studies (e.g., Feltz, 2015; Raijmakers et al., 2013) used hypothetical scenarios as well, it is not clear to what degree might judgments of morality differ when they relate to real-world cases of people who were assisted in dying. While realworld moral decisions may differ from decisions in hypothetical situations (FeldmanHall et al., 2012), most people will not actually decide about euthanasia of other people. Nevertheless, moral evaluation explored in the present study is still important, for example, during a public discussion whether to legalise euthanasia. Second, it is possible that the difference in justifiability of euthanasia between the two scenarios could have been too large. A case of a patient who does not suffer physically and whose life is not in danger could be far removed from a prototypical euthanasia scenario for many people. Previous research found that larger differences between two exemplars are more likely to lead to contrast effects, while smaller differences more likely result in assimilation effects (Mussweiler, 2003). It is therefore possible that we could have found the order effect predicted by the attitudinal slippery slope argument if we had used scenarios where justifiability of euthanasia differed less. Third, participants in the present study were laypeople who may have not given much thought to the issue of euthanasia. It is possible that the order effect could have been reduced in people who had more experience with medical settings and people who gave the issue of euthanasia more thought before taking part in the studies and who would, therefore, be more likely to have stable attitudes. However, previous research found little difference in the strength of order effects in moral judgment between professional philosophers and laypeople (Schwitzgebel \& Cushman, 2012), which suggests that the lack of expertise might not have played a large role in the present study as well. Fourth, some of the results found in the first study were not replicated in the second study. Further studies replicating the effect found in the present paper could help to evaluate which of the found effects are reliable (Open Science Collaboration, 2015). Fifth, we did not explore a long-term effect of the order of the scenarios and it is, therefore, impossible to say whether the observed attitudinal changes are only transient, or if they are more durable. Finally, the observed effects were relatively small. However, they still demonstrate that expressed attitudes towards euthanasia are malleable even by a very minimal manipulation. And although it is impossible to estimate realworld consequences of this malleability based on the current study, we could reasonably expect that influences affecting attitudes might be stronger in the real-world, where people encounter real cases rather than descriptions of hypothetical cases.

Even though we did not find support for the attitudinal slippery slope effect in the present study, it is possible that different mechanisms may lead to an occurrence of the slippery slope effect. For example, attitudinal slippery slope effects might work through habituation or routinization 
which could not have been evaluated in the present study because it took only a few minutes and presented just two euthanasia cases. However, there is currently little evidence for operation of these possible underlying mechanisms (Emanuel et al., 2016; Smith, 2005). Their further critical evaluation should help to assess general plausibility of attitudinal slippery slope effects related to euthanasia.

\section{Acknowledgements}

This research has been supported by the Internal grant of the Faculty of Arts, Charles University, ref no. FF-VG2016-79, co-awarded to Marek Vranka.

\section{Compliance with Ethical Standards}

The study was funded by the Internal grant of the Faculty of Arts, Charles University (ref no. FFVG2016-79). All procedures performed in studies involving human participants were in accordance with the ethical standards of the institutional and/or national research committee and with the 1964 Helsinki declaration and its later amendments or comparable ethical standards. Implicit informed consent was used in the study, which did not pose any risks to the participants.

\section{Conflict of interests}

The authors declare no conflict of interest associated with publication of the manuscript.

\section{References}

Andrade, E. B., \& Ariely, D. (2009). The enduring impact of transient emotions on decision making. Organizational Behavior and Human Decision Processes, 109, 1-8.

Chandler, J., Paolacci, G., Peer, E., Mueller, P., \& Ratliff, K. A. (2015). Using nonnaive participants can reduce effect sizes. Psychological Science, 26, 1131-1139.

Cohen, J., Van Landeghem, P., Carpentier, N., \& Deliens, L. (2012). Different trends in euthanasia acceptance across Europe. A study of 13 western and 10 central and eastern European countries, 1981-2008. The European Journal of Public Health, 23, 378-380.

Dierickx, S., Deliens, L., Cohen, J., \& Chambaere, K. (2015). Comparison of the expression and granting of requests for euthanasia in Belgium in 2007 vs 2013. JAMA Internal Medicine, 175, 1703-1706.

Elliott, C. (1993). Choosing Death. British Medical Journal, 306, 1075.

Emanuel, E. J. (1994). Euthanasia: Historical, ethical, and empiric perspectives. Archives of Internal Medicine, 154, 1890-1901. 
Emanuel, E. J. (2002). Euthanasia and physician-assisted suicide: a review of the empirical data from the United States. Archives of Internal Medicine, 162, 142-152.

Emanuel, E. J., Onwuteaka-Philipsen, B. D., Urwin, J. W., \& Cohen, J. (2016). Attitudes and practices of euthanasia and physician-assisted suicide in the United States, Canada, and Europe. JAMA, 316, 79-90.

Fedorikhin, A., \& Cole, C. A. (2004). Mood effects on attitudes, perceived risk and choice: Moderators and mediators. Journal of Consumer Psychology, 14, 2-12.

FeldmanHall, O., Mobbs, D., Evans, D., Hiscox, L., Navrady, L., \& Dalgleish, T. (2012). What we say and what we do: The relationship between real and hypothetical moral choices.

Cognition, 123, 434-441.

Feltz, A. (2015). Everyday attitudes about euthanasia and the slippery slope argument. In M. Cholbi \& J. Varelius (Eds.), New Directions in the Ethics of Assisted Suicide and Euthanasia (pp. 217-237). Switzerland: Springer International Publishing.

Guadagno, R. E., \& Cialdini, R. B. (2010). Preference for consistency and social influence: A review of current research findings. Social Influence, 5, 152-163.

Hall, L., Johansson, P., Tärning, B., Sikström, S., \& Deutgen, T. (2010). Magic at the marketplace: Choice blindness for the taste of jam and the smell of tea. Cognition, 117, 54-61.

Kinsella, T. D., \& Verhoef, M. J. (1993). Alberta euthanasia survey: 1. Physicians' opinions about the morality and legalization of active euthanasia. Canadian Medical Association Journal, $148,1921-1926$.

Lerner, B. H., \& Caplan, A. L. (2015). Euthanasia in Belgium and the Netherlands: On a slippery slope?. JAMA Internal Medicine, 175, 1640-1641.

Loewenstein, G. (1996). Out of control: Visceral influences on behavior. Organizational Behavior and Human Decision Processes, 65, 272-292.

Mussweiler, T. (2003). Comparison processes in social judgment: Mechanisms and consequences. Psychological Review, 110, 472-489.

O’Hara, R. E., Sinnott-Armstrong, W., \& Sinnott-Armstrong, N. A. (2010). Wording effects in moral judgments. Judgment and Decision Making, 5, 547-554.

Open Science Collaboration. (2015). Estimating the reproducibility of psychological science. Science, 349, 943.

Petrinovich, L., \& O'Neill, P. (1996). Influence of wording and framing effects on moral intuitions. Ethology and Sociobiology, 17, 145-171. 
Radbruch, L., Leget, C., Bahr, P., Müller-Busch, C., Ellershaw, J., de Conno, F., \& Vanden Berghe, P. (2016). Euthanasia and physician-assisted suicide: A white paper from the European Association for Palliative Care. Palliative Medicine, 30, 104-116.

Raijmakers, N. J., van der Heide, A., Kouwenhoven, P. S., van Thiel, G. J., van Delden, J. J., \& Rietjens, J. A. (2013). Assistance in dying for older people without a serious medical condition who have a wish to die: A national cross-sectional survey. Journal of Medical Ethics, 41, 145150 .

Schwarz, N. (2007). Cognitive aspects of survey methodology. Applied Cognitive Psychology, $21,277-287$.

Schwarz, N., \& Bless, H. (1991). Constructing reality and its alternatives: Assimilation and contrast effects in social judgment. In L. L. Martin \& A. Tesser (Eds.), The construction of social judgment, (pp. 217-245). Hillsdale, NJ: Erlbaum.

Schwarz, N., Bless, H., Strack, F., Klumpp, G., Rittenauer-Schatka, H., \& Simons, A. (1991). Ease of retrieval as information: Another look at the availability heuristic. Journal of Personality and Social psychology, 61, 195-202.

Schwitzgebel, E., \& Cushman, F. (2012). Expertise in moral reasoning? Order effects on moral judgment in professional philosophers and non-philosophers. Mind \& Language, 27, 135-153.

Smith, S. W. (2005). Evidence for the practical slippery slope in the debate on physician-assisted suicide and euthanasia. Medical Law Review, 13, 17-44.

Stewart, N., Ungemach, C., Harris, A. J., Bartels, D. M., Newell, B. R., Paolacci, G., \& Chandler, J. (2015). The average laboratory samples a population of 7,300 Amazon Mechanical Turk workers. Judgment and Decision making, 10, 479-491.

Strack, F., Martin, L. L., \& Schwarz, N. (1988). Priming and communication: Social determinants of information use in judgments of life satisfaction. European Journal of Social Psychology, 18, 429-442.

Volokh, E. (2003). The mechanisms of the slippery slope. Harvard Law Review, 116, 10261137.

Vrakking, A. M., van der Heide, A., Looman, C. W., van Delden, J. J., Onwuteaka-Philipsen, B. D., van der Maas, P. J., \& van der Wal, G. (2005). Physicians' willingness to grant requests for assistance in dying for children: A study of hypothetical cases. The Journal of Pediatrics, 146, 611-617.

Vranka, M. A., \& Bahník, Š . (2016). Is the Emotional Dog Blind to Its Choices? Experimental Psychology, 63(3), 180-188. 
World Health Organization (2004). A Glossary of Terms for Community Health Care and Services for Older Persons. Kobe: WHO.

Wilson, T. D., \& Hodges, S. (1992). Attitudes as temporary constructions. In L. Martin \& A. Tesser (Eds.), The construction of social judgements, 37-65. New York: Springer.

Wiegmann, A., Okan, Y., \& Nagel, J. (2012). Order effects in moral judgment. Philosophical Psychology, 25(6), 813-836. 